\title{
The Inference of Structure in Images using Multi-local Quadrature Filters.
}

\author{
K.Langley \\ Department of Psychology, \\ University College London, \\ London, WC1E 6BT. U.K.
}

\author{
T.J.Atherton \\ Dept. Computer Science, \\ University of Warwick, \\ Coventry, CV4 7AL. U.K.
}

\begin{abstract}
Two techniques are presented for corner detection. First, a band of filters are applied with equal radial spatial frequency, but different orientation preferences locally in the image domain. From the energy response, a linear Fourier transform is taken to give confidence measures of both "cornerness" and "edgeness. Second, we consider a multi-local spatial separation of filters that lie on a constant radius from a point of interest. This second stage of processing allows a wider classification of image structure. As a result, we infer the presence of line end points, " $L ", " T ", " Y$ " and " $X "$ junctions using epistemic probabilities. The results are indicative of a relationship between Fourier and Spatial domain models of filtering.
\end{abstract}

\section{Introduction}

Much attention has been given to the detection of edges in low-level picture processing to facilitate a symbolic description of image detail. Noble [4], however, has pointed out that conventional edge detecting operators fail to locate both corners and junctions. Li et al[3] have proposed a mechanism by which it is possible to overcome the destruction of edge connectivity associated with " $\mathrm{T}$ " junctions using the Canny edge detector but this scheme is restricted to edges and does not consider lines. Harris[1], has designed a corner detector based upon the tensor representation of the local image using Gaussian derivative operators. We consider the application of Gabor functions for corner detection but we differ from Harris by applying filter kernels in quadrature. This representation is independent on the phase of the signal and therefore has no direct bias between lines and edges.

\section{Corner detection}

We first consider the detection of a corner feature by applying an ordered arrangement of orientation and spatial frequency bandlimited filters. The technique is based upon the observation that at an edge or line a single orientation is present, whilst at a corner, two orientations can be recorded. The filter is based upon the Gabor function and 
applied in quadrature. The filter $(\vec{\Psi}(x, y ; u, v))$ has a standard form with orientational $\left(\theta_{i}\right)$ and spatial frequency $\left(r_{o}\right)$ described by:

$$
\vec{\Psi}_{i}(x, y ; u, v)=k_{1} \exp \left[-\frac{x^{\prime 2}}{4 \sigma_{x}^{2}}-\frac{y^{\prime 2}}{4 \sigma_{y}^{2}}\right] \exp \left[j<\vec{u}_{i}, \vec{x}^{\prime}>\right] 1 \leq i \leq N
$$

where $\sqrt{u_{i}^{2}+v_{i}^{2}}=r_{o}$ resolves the modulation frequency of the filter into horizontal $\left(u_{i}\right)$ and vertical $\left(v_{i}\right)$ spatial frequency components. Additionally:

$$
\vec{x}^{\prime}=\left[\begin{array}{l}
x^{\prime} \\
y^{\prime}
\end{array}\right]=\left[\begin{array}{cc}
\cos \theta_{i} & \sin \theta_{i} \\
-\sin \theta_{i} & \cos \theta_{i}
\end{array}\right]\left[\begin{array}{l}
x \\
y
\end{array}\right] \text { and } \vec{u}_{i}=\left[\begin{array}{c}
u_{i} \\
v_{i}
\end{array}\right]=\left[\begin{array}{c}
r_{o} \cos \theta_{i} \\
r_{o} \sin \theta_{i}
\end{array}\right]
$$

which defines the nature of each oriented filter in the spatial domain. The arrangement of filters is also shown in figure 1. Notice because of the one-sided nature of the Hilbert transform, we restrict analysis to $\pi$ radians in the Fourier domain.

We operate on the image $(I(x, y))$ by:

$$
E_{i}(x, y ; u, v)=\left|\vec{\Psi}\left(x, y ; u_{i}, v_{i}\right) * I(x, y)\right|
$$

where we apply $i=1$ to $N$ orientationally selective filters at each pixel. Equation (1) represents an energy function mapped onto an interval on the real line $[0, \pi]$. At an image region with frequency components in two orthogonal directions such as a corner, the energy responses of the filters will show peaks in the filters separated by $\frac{\pi}{2}$ radians.

To examine this in more detail, we consider a frequency domain model describing the behaviour of the linear filter in the presence of corners. The model assumes that locally, a line/edge can be approximated by a line of unknown orientation passing through the origin of the Fourier domain (thus our model does not take into account dispersion in the frequency domain). In the case of a corner, there are two such lines passing through the origin of the frequency domain separated by right angles (fig. 1). These pair of lines are represented by the unit vectors $\overrightarrow{\hat{n}}$ and $\overrightarrow{\hat{n}}_{\perp}$ respectively whose orientation we wish to estimate.

We begin by defining an orientation at a corner as the orientation which minimises the energy function $Q_{c}(\theta)$, where:

$$
Q_{c}(\theta)=\sum_{i=0}^{N} E_{i}^{2}(x, y ; u, v) d_{1 i}^{2} d_{2 i}^{2}=\sum_{i=0}^{N} E_{i}^{2}(x, y ; u, v)\left[\left.\left.\left|<\vec{r}_{i}, \overrightarrow{\hat{n}}^{2}\right\rangle\right|^{2}\left|<\vec{r}_{i}, \overrightarrow{\hat{n}}_{\perp}\right\rangle\right|^{2}\right]
$$

and $\overrightarrow{\mathbf{r}}_{i}=r_{o}\left[\cos \theta_{i}, \sin \theta_{i}\right]^{T}$ represents the position vector of the $i$ th filter in the frequency domain. In figure 1 , the lengths $d_{1 i}$ and $d_{2 i}$ represent the lengths whose squared products we wish to minimise. We minimise the product since we have defined a local maxima in the energy function $E(x, y ; u, v)$ as the orientation of one of the dominant frequency components that we wish to measure. From our model, this orientation is orthogonal to, and therefore independent of, the second signal present. The angle $\theta_{c}$ that minimises the function $Q(\theta)$ is:

$$
\tan 4 \theta_{c}=\frac{\sum_{i=0}^{N} E_{i}^{2}(x, y ; u, v) \sin 4 \theta_{i}}{\sum_{i=0}^{N} E_{i}^{2}(x, y ; u, v) \cos 4 \theta_{i}}
$$

Equation (3), gives an estimation of the orientation of a corner in the image. However, 

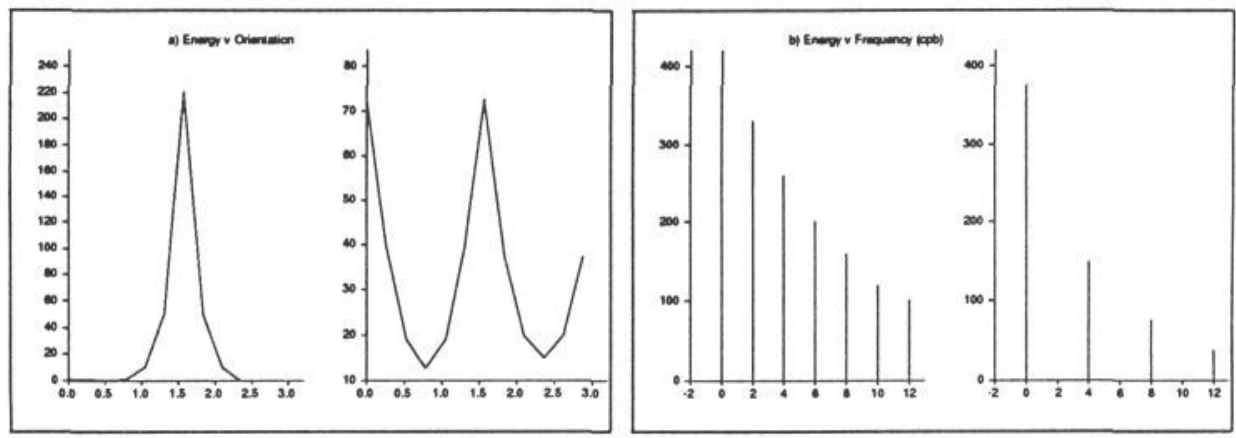

Figure 2: a) Energy response from a band of 2-D Gabor filters to an ideal edge (left) and corner (right). (b) The discrete Fourier transform taken from the energy responses in a) in cycles per band (cpb).

In figure $2 \mathrm{a}$ we show the function $E(x, y ; u, v)$ taken at an ideal corner and edge, with a filter envelope aspect ratio of 0.65 in favour of orientation. Figure $2 \mathrm{~b}$ shows the magnitude of the linear Fourier transform taken from the energy responses in figure $2 \mathrm{a}$.

In the presence of two-orthogonal signals the Fourier energy component primarily used for edge detection is small by comparison to the Fourier energy response at corners. However, the converse is not the case, where in the case of an edge/line, there is significant energy at all frequencies. This is because the energy response for an edge/line more closely resembles a gaussian distribution than sinusoidal function which our model assumes. At a corner, however, the presence of two dominant energy peaks suppresses the fundamental frequency which we would normally examine as a measure of edge/line probability. To disambiguate, a further constraint is required to identify regions that exhibit two-orthogonal signals:

$$
C_{c}(x, y)=P_{c}(x, y)-P_{c}(x, y)
$$

where $C_{c}(x, y)$ now expresses a corner confidence measure or epistemic probability. Because corner detection involves an examination of a higher frequency from local Energy responses, it is necessary to apply at least twice the number of filters by comparison to edge/line detection only.

\section{Higher levels of processing}

As a consequence of processing directionally selective filters at a single point in the image domain it is not possible to classify the structure of local junctions using this representation. Thus, an " $L$ " or " $\mathrm{X}$ " junction will be inseparable. At an ideal image corner, each oriented filter can only integrate directional energy over half its spatial extent in the image domain which contributes to noise sensitivity of local corner detection. To facilitate the examination of local image structure in more detail and improve noise sensitivity, we now consider processing the response from filters that are separated by a constant radius but different orientation in the spatial domain. This may be considered as the spatial analog of the frequency domain model considered previously. 


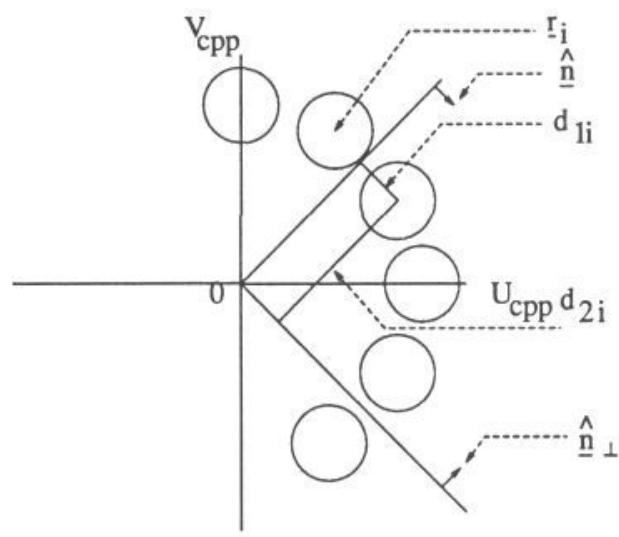

Figure 1: A circular band of $R_{i}$ filters considered in the 2-D Fourier domain in cycles per pixel (cpp). The circles indicate the frequency domain bandwidth of each filter.

because we have applied local operations it is only possible to distinguish between the orientation of corner features separated by $\frac{\pi}{4}$ radians. Equation (3) extends the result of Knutsson et al [2], who showed that edge orientation may be obtained from a set of orientationally selective filters by applying the Willsky error measure on a circle [5]. Using the least squares estimation that we have shown above, and applied to the case of an image function with a single dominant orientation we also obtain the orientation $\left(\theta_{e}\right)$ by minimising:

$$
Q_{e}(\theta)=\sum_{i=0}^{N} E_{i}^{2}(x, y ; u, v)\left|<\vec{r}_{i}, \overrightarrow{\hat{n}}>\right|^{2}
$$

The estimation of orientation then reduces to the familier form:

$$
\tan 2 \theta_{e}=\frac{\sum_{i=0}^{N} E_{i}^{2}(x, y ; u, v) \sin 2 \theta_{i}}{\sum_{i=0}^{N} E_{i}^{2}(x, y ; u, v) \cos 2 \theta_{i}}
$$

Equations (3) and (4) indicate that the orientation at both corner and edge locations can be described by the phase of the Fourier components present in the energy function taken from equation (1). The energy responses taken from the filters located at an ideal edge and corner are shown in figure 2 . The periodicity of $\frac{\pi}{2}$ and $\frac{\pi}{4}$ is clear.

To derive a probabilistic measure associated with the estimates of the edge $\left(P_{e}\right)$ and corner $\left(P_{c}\right)$ orientations we apply:

$$
\begin{aligned}
& P_{e}=\frac{\left[\sum_{i=0}^{N} E_{i}^{2}(x, y ; u, v) \cos 2 \theta_{i}\right]^{2}+\left[\sum_{i=0}^{N} E_{i}^{2}(x, y ; u, v) \sin 2 \theta_{i}\right]^{2}}{\left[\sum_{i=0}^{N} E_{i}^{2}(x, y ; u, v)\right]^{2}} \\
& P_{c}=\frac{\left[\sum_{i=0}^{N} E_{i}^{2}(x, y ; u, v) \cos 4 \theta_{i}\right]^{2}+\left[\sum_{i=0}^{N} E_{i}^{2}(x, y ; u, v) \sin 4 \theta_{i}\right]^{2}}{\left[\sum_{i=0}^{N} E_{i}^{2}(x, y ; u, v)\right]^{2}}
\end{aligned}
$$

which merely requires an examination of the magnitudes of the Fourier components relative to the d.c energy present in the response taken from the band of filters. 
In this scheme we are primarily interested in local edges/lines which requires a smaller number of local filters and reduces the computational burden.

We obtain the orientation and probabilistic description of the local image structure using a similar model in the spatial domain to that considered in the frequency domain:

$$
\tan n \theta=\frac{\sum_{i=0}^{N} P_{e}\left(x_{i}-x_{o}, y_{i}-y_{o}\right) \sin n \theta_{i}}{\sum_{i=0}^{N} P_{e}\left(x_{i}-x_{o}, y_{i}-y_{o}\right) \cos n \theta_{i}}
$$

and $P_{n}(x, y)=$

$$
G(n)\left[\frac{\sum_{i=0}^{N} P_{e}\left(x_{i}-x_{o}, y_{i}-y_{o}\right) W_{i} \cos n \theta_{i}+\sum_{i=0}^{N} P_{e}\left(x_{i}-x_{o}, y_{i}-y_{o}\right) W_{i} \sin n \theta_{i}}{n}\right]
$$

with $\left(x_{i}-x_{o}\right)^{2}+\left(y_{i}-y_{o}\right)^{2}=R_{o}^{2}$ and $\mathrm{n}=1,2,3,4$ represents the Fourier energies where we expect line end points, lines/edges, "Y" junctions, and " $\mathrm{X}$ " junctions to give dominant responses respectively. $R_{o}$ represents the magnitude of the spatial separation of filters in the image domain. The added weightings $W$ and $G$ are discussed below. Notice that the sum of lines/edges are now used to normalise equation (9) in replace of the energy sum used in the local technique considered previously.

One consequence of the spatial circle which holds an analogy to dispersion in the spatio-temporal domain, lies the restriction that the local orientation of energy passing through each spatially displaced filter must be expected to project through to the origin of the cluster of filters. To ensure that this is the case we use a form of inner product weighting based upon the Willsky error measure that results because of the double angle representation of orientation for edges/lines:

$$
\left.W_{i}=\left(\frac{1}{2}\right)^{m}\left[1+\cos \phi_{d}\right)\right]^{m}
$$

where $m$ is an orientation weighting that restricts the orientation difference $\phi_{d}$ between the measured orientation and the orientation of the spatial filter relative to the center of the filter cluster (note in the latter case the orientation of the spatially displaced filter point is also defined in the double angle form).

\subsection{The detection of substructures}

One difficulty with "L" and " $T$ " junctions lies with the fact that there is no unique Fourier energy in terms of the representation considered. In the case of the " $L$ " junction, the Fourier domain representation exhibits energy at both $n=4$ and $n=1$ frequencies. Because of the nonorthogonality of this type of structure it is necessary to consider the summation of edge/line probabilities over the spatial cluster of filters, where for "L" junction we would expect the summation of edge/line probability to be approximately two. This leads to the inclusion of an additional Gaussian weighting:

$$
G(n)=\exp \left[-\frac{\left(\sum_{i=0}^{N} P_{e}\left(x_{i}-x_{o}, y_{i}-y_{o}\right)-n\right)^{2}}{k_{2}}\right]
$$

where $k_{2}$ is a constant and $n$ refers to an expected sum of edge/line probabilities (for the " $L$ " junction we have used $n=1.5: 1.0<n<2.0$ ). The epistemic probability of an " $\mathrm{L}$ " junction is then taken using:

$$
C_{L}(x, y)=\left[P_{4}(x, y)+P_{1}(x, y)\right] G(1.5)
$$




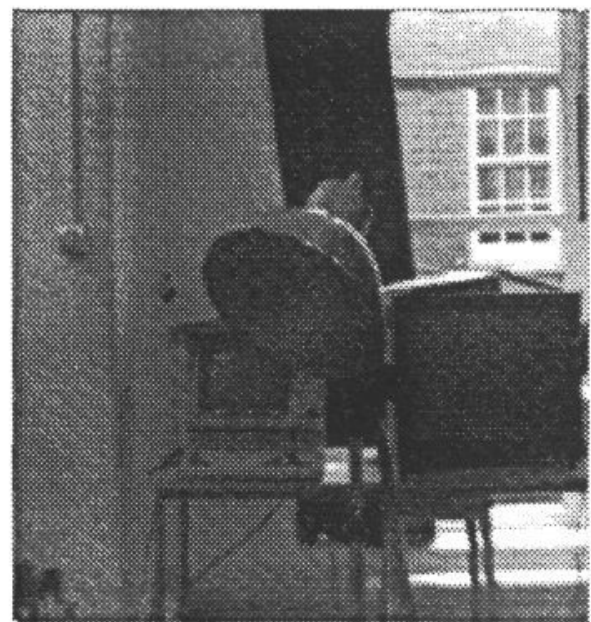

Figure 3: (a) Left Original "room" image. (b) Right Local corner detection probability.

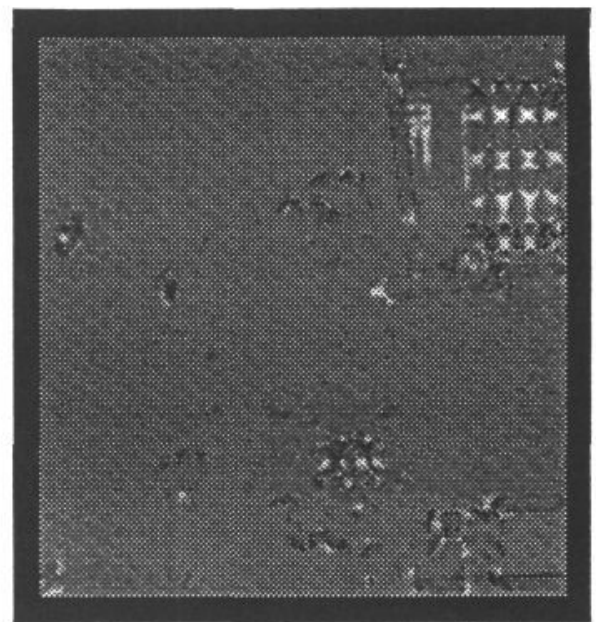

which will be larger in magnitude than the probabilities of the other local image structures under consideration.

\section{Results}

Results are presented to a single real image for both local and multi-local corner detection. In the case of local corner detection, high corner confidences were recorded surrounding the window frames, with weaker responses at image regions with poorer contrast. However, high corner probabilities have been recorded owing to the corner features present with the "brick structure" of the outside wall (fig. $3 \mathrm{~b}$ ). The intensity changes within these structures is very small, and therefore indicative of the noise sensitivity of local operators. Figures $(4 \mathrm{a}$ and $4 \mathrm{~b})$ represent the normalised probability image for both " $\mathrm{L}$ " and " $\mathrm{X}$ " junctions using the spatial cluster of filters applied multi-locally. The ability to now distinguish the nature of the junction is clear.

\section{Conclusion}

We have shown in principle how localised 2D-Gabor filters may be applied to the problem of corner detection. However, this technique requires a large number of local filters and is noise sensitive particularly at " $L$ " junctions because each filter can only integrate of half its spatial extent in the image domain. By processing the epistemic line/edge probabilities on the spatial circle, we note that it is now possible to classify and describe the local structure of the image in greater detail, as well as decreasing both the noise sensitivity of corner detection and the number of filters that applied locally. More interestingly, it is noted that the analytic models considered in the frequency domain can equally be applied in the spatial domain. 

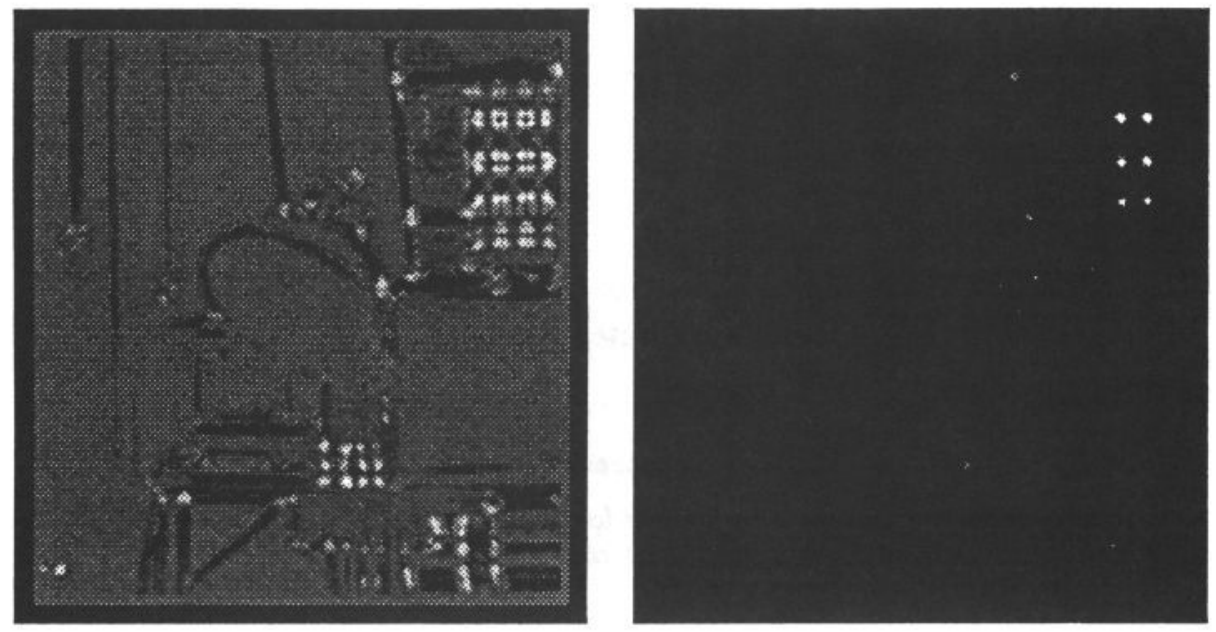

Figure 4: (a) Left Probability of an "L" junction: edge probability subtracted for clarity. (b) Right Probability of an " $X$ " junction.

\section{References}

[1] Harris.C and Stephens.M. A combined corner and edge detector. Proc. 4th Alvey vision conf.,147-151, 1988.

[2] Knutsson.H., Wilson.R.G., and Granlund.G. Estimating the orientation of local anistropic 2-d signals. IEEE. A.S.S.P.,Florida, 234-239, 1983.

[3] Du Li, Sullivan.G.D, and Baker.K.D. Edge detection at junctions. 5th Alvey vision conf.,121-127, 1989.

[4] Noble.A. Finding corners. 3rd Alvey Vision Conference,267-274, 1987.

[5] Willsky.A.S. Fourier series and estimation on the circle with applications to synchronous communication. part 1:analysis. IEEE Trans.Info.Theory, IT-20,5, 1974. 